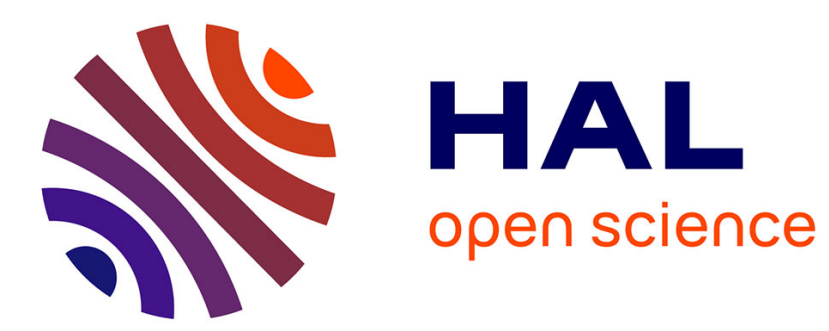

\title{
ELLIPTIC EQUATIONS WITH ABSORPTION IN A HALF-SPACE
}

Jorge García-Melián, A Quaas, B Sirakov

\section{To cite this version:}

Jorge García-Melián, A Quaas, B Sirakov. ELLIPTIC EQUATIONS WITH ABSORPTION IN A HALF-SPACE. 2015. hal-01111679v2

\section{HAL Id: hal-01111679 \\ https://hal.science/hal-01111679v2}

Preprint submitted on 22 Oct 2015

HAL is a multi-disciplinary open access archive for the deposit and dissemination of scientific research documents, whether they are published or not. The documents may come from teaching and research institutions in France or abroad, or from public or private research centers.
L'archive ouverte pluridisciplinaire HAL, est destinée au dépôt et à la diffusion de documents scientifiques de niveau recherche, publiés ou non, émanant des établissements d'enseignement et de recherche français ou étrangers, des laboratoires publics ou privés. 


\title{
ELLIPTIC EQUATIONS WITH ABSORPTION IN A HALF-SPACE
}

\author{
J. GARCÍA-MELIÁN, A. QUAAS AND B. SIRAKOV
}

\begin{abstract}
We give a necessary and sufficient condition, in the spirit of the classical works by Keller and Osserman, for the elliptic equation $\Delta u=f(u)$ to have a solution in a half-space of $\mathbb{R}^{N}$. The function $f$ is supposed to be nondecreasing and nonnegative, and we are interested in solutions whose range is where $f>0$. The possibility of obtaining such a necessary and sufficient condition has been an open question for a long time.
\end{abstract}

\section{INTRODUCTION AND RESULTS}

The aim of this work is to analyze the existence and nonexistence of solutions of the problem

$$
\Delta u=f(u) \text { in } \mathbb{R}_{+}^{N},
$$

where the function $f$ is continuous, nonnegative and nondecreasing on $\mathbb{R}$, and $\mathbb{R}_{+}^{N}$ denotes the half-space $\mathbb{R}_{+}^{N}=\left\{x \in \mathbb{R}^{N}: x_{N}>0\right\}$. We also study the existence of subsolutions of this problem, that is, solutions of the inequality

$$
\Delta u \geq f(u) \text { in } \mathbb{R}_{+}^{N} .
$$

The classical papers by Osserman [15] and Keller [12] have generated a tremendous amount of work on this type of equations, most of which has been devoted to the study of entire solutions or of explosive solutions in a bounded domain. As already explained in [15] (see p. 1644 in that paper) it is meaningful to search for solutions whose range is in the set where $f$ is strictly positive, since without this assumption the solution set can indeed be very large and variable. Thus, in the following we will consider such solutions only. Note that since $f$ is nondecreasing and nonnegative, we have $\{t: f(t)>0\}=\left(t_{0}, \infty\right)$ for some $t_{0} \geq-\infty$. Up to a simple transformation we can assume that $t_{0}=0$ or $t_{0}=-\infty$, in other words, either $f(t)>0$ for $t>0$ and $u>0$, or $f>0$ on $\mathbb{R}$ and there is no sign restriction on $u$.

In particular, it is known from [15] that the equation $\Delta u=f(u)$ does not have solutions in the whole space $\mathbb{R}^{N}$ if and only if

$$
\int_{1}^{\infty} \frac{d s}{\sqrt{F(s)}}<\infty
$$

where $F(s)=\int_{0}^{s} f(t) d t$ is a primitive of $f$ (this condition is nowadays usually referred to as the Keller-Osserman condition). On the other hand, (explosive) solutions in a bounded domain exist if and only if $(\mathrm{KO})$ holds. It is outside the scope of this short paper to attempt a reasonably full bibliography for the many developments that followed [15] and [12, we refer for instance to the surveys [9] and [17], where a large set of references can be found. 
However, to the best of our knowledge and maybe somewhat surprisingly, a study of the problem is not available for proper unbounded subdomains of $\mathbb{R}^{N}$ such as a half-space (except for a comment on nonexistence of radial solutions in Remark 1.6 of [14). Elliptic equations on a half-space are both intrinsically important and appear in the study of other problems, such as the study of existence of a priori bounds for solutions through the Gidas-Spruck blow-up method. Half-spaces and cones also naturally appear in the study of boundary behaviour of solutions of elliptic equations in Lipschitz domains.

We observe that the solvability of $(\mathrm{P})$ has been extensively studied in the particular case when the function is supposed to satisfy a boundary condition at $\partial \mathbb{R}_{+}^{N}=$ $\left\{x \in \mathbb{R}^{N}: x_{N}=0\right\}$, such as $u=0$ on this set. We refer for instance to [1], [2], [3], [4], [5], [13, and the references in these works.

In this paper we provide a necessary and sufficient condition for $(\mathrm{P})$ to have a solution. This is the content of the following theorem. Throughout the paper, an existence statement will mean that (P) has a strong (or even classical, if $f$ is supposed to be Hölder continuous) solution, whereas a non-existence statement will mean (P) does not even have solutions in any weak (such as weak-Sobolev or viscosity) sense which leaves valid the comparison principle.

Note that since the existence of a solution in $\mathbb{R}^{N}$ implies existence in any subdomain of $\mathbb{R}^{N}$, we only need to study the problem under the hypothesis (KO).

Theorem 1. Suppose (KO) holds. Then equation $(P)$ has a solution if and only if

$$
\left\{\begin{array}{l}
\int_{0}^{1} \frac{d s}{\sqrt{F(s)}}=\infty \text { if } t_{0}=0, \text { or } \\
\int_{-\infty}^{-1} f(s) d s<\infty \text { if } t_{0}=-\infty
\end{array}\right.
$$

If this hypothesis is not satisfied, even subsolutions do not exist.

We stress that this theorem completely characterizes the existence of solutions and subsolutions of $(\mathrm{P})$, and thus represents a counterpart for a half-space of the results of Keller and Osserman for bounded domains or the whole space.

At this point a discussion on the possibility of extending the above theorem to more general unbounded domains is in order. The situation turns out to be very different depending on whether $t_{0}=0$ or $t_{0}=-\infty$.

First, if $t_{0}=0$ then the first condition in (HS) actually guarantees the existence of a positive solution in any subdomain of $\mathbb{R}^{N}$ whose closure is not the whole space. Note that this condition is what ensures the validity of the strong maximum principle for nonnegative solutions of $\Delta u=f(u)$, by the well-known result of Vázquez ([18], see also [16]). On the other hand, if (HS) fails when $t_{0}=0$ then positive solutions do not exist in any sufficiently "fat" subdomain of $\mathbb{R}^{N}$. Thus, the result in Theorem 1 remains the same if $t_{0}=0$ and the half-space is replaced by any strictly proper subdomain of $\mathbb{R}^{N}$ which contains balls of arbitrary radius - such as, for instance, a nontrivial cone in $\mathbb{R}^{N}$ (see Theorem 2 below). 
Second, if $t_{0}=-\infty$ then the condition in (HS) appears strongly related to the specific geometry of the half-space. If a different cone in $\mathbb{R}^{N}$ is considered, then this condition no longer characterizes the solvability of $\Delta u=f(u)$. For instance, the function $\underline{u}=-x_{1}^{\alpha} x_{2}^{\alpha}$ is a solution of the inequality $\Delta u \geq c|u|^{\beta}$ in the quarter-space $\left\{x_{1}>0, x_{2}>0\right\}$ for any $\beta \in(-1,0)$ (and so $f(u)=|u|^{\beta}$ does not satisfy (HS)), provided $\alpha=\frac{1}{1-\beta}$ and $c=2 \alpha(1-\alpha)$. Hence if $f: \mathbb{R} \rightarrow(0, \infty)$ is increasing and $f(u) \leq|u|^{\beta}$ for $u<0$ then a solution of $\Delta u=f(u)$ in the quarterspace exists since $\underline{u}$ is a subsolution, $\bar{u}=0$ is a supersolution, and they are ordered. Currently we do not know how to obtain a necessary and sufficient condition for existence of solutions of $(\mathrm{P})$ in a cone different from $\mathbb{R}^{N}$ or $\mathbb{R}_{+}^{N}$, in the case $t_{0}=-\infty$. We believe this is an interesting open problem.

It is to be stressed that, while the existence and nonexistence of (sub-)solutions of $(\mathrm{P})$ in the whole space depends only on the behavior at infinity of $f$, the result above shows that the behavior at $t_{0}=\inf \{t: f(t)>0\}$ of $f$ turns out to be important when a proper unbounded subdomain is considered.

\section{ProOFS}

In this section we will prove Theorem 1. The proofs depend on the comparison principle, together with the resolution of some auxiliary problems. We recall that the monotonicity of $f$ implies the equation is proper, thus satisfies the weak comparison principle, in the sense that a subsolution and a supersolution which are ordered on the boundary of any bounded domain are also ordered throughout the domain. Also, Perron's method applies and yields the existence of a solution between any given ordered subsolution and a supersolution (see for instance [8] for classical or weak-Sobolev solutions and [6] for viscosity solutions).

Before giving the proof we briefly recall the construction of an entire (radial) solution of $\Delta u=f(u)$ when the condition $(\mathrm{KO})$ is not satisfied. Fix a real number $v_{0}$ such that $f\left(v_{0}\right)>0$ and consider the following ordinary differential equation for a function $v=v(r)$,

$$
\left\{\begin{array}{l}
v^{\prime \prime}+\frac{N-1}{r} v^{\prime}=f(v), \quad r>0 \\
v(0)=v_{0}, v^{\prime}(0)=0 .
\end{array}\right.
$$

Since $f$ is continuous, problem $(2.1)$ has at least one $C^{2}$ solution $v$ defined in a maximal interval of existence $[0, R)$ for some $R>0$. Since $\left(r^{N-1} v^{\prime}\right)^{\prime}=r^{N-1} f(v) \geq 0$ we have $r^{N-1} v^{\prime} \geq 0$ so that $v$ is increasing and therefore $v^{\prime \prime} \leq f(v)$, by (2.1). We can multiply this inequality by $v^{\prime}$, write the result as $\left(\left(v^{\prime}\right)^{2}\right)^{\prime} \leq(2 F(v))^{\prime}$, and integrate to get

$$
\int_{v_{0}}^{v(r)} \frac{d t}{\sqrt{2 F(t)}} \leq r
$$

for every $r \in(0, R)$, which implies, since (KO) does not hold, that $R$ is infinite. Thus for any $x_{0} \in \mathbb{R}^{N}$ the function $u(x)=v\left(\left|x-x_{0}\right|\right)$ is a positive solution of $\Delta u=f(u)$ in $\mathbb{R}^{N}$. The minimum of $u$ is attained at $x_{0}$ and its value is $v_{0}$. 
On the other hand, when $(\mathrm{KO})$ is satisfied, the solution of (2.1) blows up at a finite $R$; indeed, since the equation in 2.1 can also be written as

$$
v^{\prime}(r)=\int_{0}^{r}\left(\frac{s}{r}\right)^{N-1} f(v(s)) d s,
$$

from the monotonicity of $f$ and $v$ we have $v^{\prime}(r) \leq \frac{r}{N} f(v(r))$, so that

$$
v^{\prime \prime} \geq \frac{1}{N} f(v) .
$$

Multiplying by $v^{\prime}$ as above we get

$$
\int_{v_{0}}^{v(r)} \frac{d t}{\sqrt{F(t)-F\left(v_{0}\right)}} \geq \frac{1}{\sqrt{N}} r
$$

for every $r \in(0, R)$, which implies that $R$ is finite, by $(\mathrm{KO})$. Thus $u(x)=v\left(\left|x-x_{0}\right|\right)$ solves $\Delta u=f(u)$ in $B_{R}\left(x_{0}\right)$ with $v=+\infty$ on $\partial B_{R}\left(x_{0}\right)$ (throughout the paper, $B_{r}\left(x_{0}\right)$ stands for the ball with radius $r$ centered at $x_{0}$; we write $B_{r}$ when $\left.x_{0}=0\right)$.

Proof of Theorem 1, case $t_{0}=0$.

Existence. Assume that (HS) holds, that is, the Vázquez condition for the strong maximum principle $\int_{0} F^{-1 / 2}=\infty$ is verified. Take any $x_{0} \in \mathbb{R}^{N}$ and $r>0$ such that $B_{r}\left(x_{0}\right) \subset\left\{x_{N}<0\right\}$. Since $f(0)=0$ and $f(\delta)>0$ for some small $\delta>0$, the functions $\underline{u}=0$ and $\bar{u}=\delta$ are respectively a subsolution and a supersolution for the problem

$$
\left\{\begin{array}{cll}
\Delta u=f(u) & \text { in } & B_{R}\left(x_{0}\right) \backslash B_{r}\left(x_{0}\right) \\
u=\delta & \text { on } & \partial B_{r}\left(x_{0}\right) \\
u=0 & \text { on } & \partial B_{R}\left(x_{0}\right) .
\end{array}\right.
$$

Hence there exists a solution $u_{R}$ of this problem such that $0 \leq u_{R} \leq \delta$. By standard elliptic estimates (see for instance [11, Theorem 9.13) the sequence $\left\|u_{R}\right\|_{W^{2, p}(K)}$ is bounded independently of $R$ in any fixed compact set $K$ of $\mathbb{R}^{N} \backslash B_{r}\left(x_{0}\right)$, for any $p<\infty$. Hence we can extract a subsequence which converges strongly in $C^{1, \alpha}(K)$ and weakly in $W^{2, p}(K)$ to a function $u$. Thus, by a diagonal argument, taking larger and larger compacts $K$, we get a sequence $R_{n} \rightarrow \infty$ such that $u_{R_{n}} \rightarrow u$ in $W_{\text {loc }}^{2, p}\left(\mathbb{R}^{N} \backslash B_{r}\left(x_{0}\right)\right)$. Passing to the limit we see that $u$ is a nonnegative solution to the equation $\Delta u=f(u)$ in $\mathbb{R}^{N} \backslash B_{r}\left(x_{0}\right)$. This solution does not vanish identically by the boundary condition $u=\delta$ on $\partial B_{r}\left(x_{0}\right)$. Since the strong maximum principle holds (see [18]) we obtain that $u$ is strictly positive in $\mathbb{R}^{N} \backslash B_{r}\left(x_{0}\right) \supset \mathbb{R}_{+}^{N}$.

Nonexistence. As we recalled above, the Keller-Osserman condition (KO) implies that for each $v_{0}=\varepsilon>0$ there is a finite number $R_{\varepsilon}$ such that the problem $\Delta w=$ $f(w)$ has a solution in each ball with radius $R_{\varepsilon}$, which blows up at the boundary of the ball, and has value $\varepsilon$ at the center of the ball.

Assume problem $(\mathrm{P})$ has a positive solution $u$. Take a (small) number $\delta>0$ and fix any ball $\hat{B}=B_{R_{\varepsilon}+\delta}\left(x_{0}\right) \subset \mathbb{R}_{+}^{N}$. We claim that $u<2 \varepsilon$ in $B_{\delta}\left(x_{0}\right)$. In fact, if we had $u(\bar{x}) \geq 2 \varepsilon$ for some $\bar{x} \in B_{\delta}\left(x_{0}\right)$, we could define $w(x)=v(|x-\bar{x}|)$, where $v$ is the solution of $(2.1)$ with $v(0)=\varepsilon$, and use the comparison principle in the 
ball $B_{R_{\varepsilon}}(\bar{x}) \subset R_{+}^{n}$ to get $u \leq w$ in this ball. However, this is a contradiction since $u(\bar{x}) \geq 2 \varepsilon$ while $w(\bar{x})=v(0)=\varepsilon$.

Finally, we claim that the failure of (HS) implies that there exists a supersolution $z$ of $(\mathrm{P})$ in $B_{\delta}\left(x_{0}\right)$ with $z=2 \varepsilon$ on $\partial B_{\delta}\left(x_{0}\right)$ and $z=0$ on $B_{\delta / 2}\left(x_{0}\right)$ (a dead core). By using the comparison principle again, this time in $B_{\delta}\left(x_{0}\right)$, we find that $u \leq 2 \varepsilon=z$ on $\partial B_{\delta}\left(x_{0}\right)$ implies $u \leq z=0$ in $B_{\delta / 2}\left(x_{0}\right)$, contradicting the fact that $u$ is positive.

Hence to conclude the proof of the theorem only this last claim remains to be proved. We will find a radial super-solution $z$ such that $z(x)=\beta\left(\left|x-x_{0}\right|\right)$, and $\beta$ is a real function which satisfies the ODE

$$
\beta^{\prime \prime}+\frac{N-1}{r} \beta^{\prime} \leq f(\beta) \text { in }(0, \delta),
$$

and is such that $\beta=0$ on $(0, \delta / 2)$ and $\beta(\delta)=2 \varepsilon$. For this purpose it suffices to find a solution in $(\delta / 2, \delta)$ such that $\beta(\delta / 2)=0, \beta^{\prime}(\delta / 2)=0$ and $\beta(\delta)=2 \varepsilon$, and then extend this solution as zero in $(0, \delta / 2)$.

To find $\beta$ we assume $N \geq 3$ and perform the change of variables $s=\frac{1}{N-2} r^{2-N}$, $\tilde{\beta}(s)=\beta(r)$ (a similar argument can be made in the case $N=2$ with the change of variables $s=\log r$; we will skip the details) and obtain the one-dimensional problem

$$
\left\{\begin{array}{l}
\tilde{\beta}^{\prime \prime} \leq c s^{-\gamma} f(\tilde{\beta}) \quad \text { in }\left(s_{0}, s_{1}\right) \\
\tilde{\beta}\left(s_{0}\right)=\varepsilon, \tilde{\beta}\left(s_{1}\right)=\tilde{\beta}^{\prime}\left(s_{1}\right)=0,
\end{array}\right.
$$

where $\gamma=\frac{2(N-1)}{N-2}, c=(N-2)^{-\gamma}, s_{0}=\delta^{2-N} /(N-2)$ and $s_{1}=2^{N-2} s_{0}$. Observe that $\gamma>2$.

Since the integral $\int_{0} F^{-1 / 2}$ is convergent we can find adequately small $\varepsilon>0$ and $\delta>0$ such that

$$
\int_{0}^{\varepsilon} \frac{d s}{\sqrt{2 F(s)}}=\sqrt{c s_{1}^{-\gamma}}\left(s_{1}-s_{0}\right)=\tilde{c} \delta^{\tilde{\gamma}}
$$

where $\tilde{c}, \tilde{\gamma}$ are positive constants which depend only on the dimension $N$. Thus we can define implicitly the function $\tilde{\beta}$ through the equality

$$
\int_{\tilde{\beta}(t)}^{\varepsilon} \frac{d s}{\sqrt{2 F(s)}}=\sqrt{c s_{1}^{-\gamma}}\left(t-s_{0}\right)
$$

for $t \in\left(s_{0}, s_{1}\right)$. Therefore $\tilde{\beta}$ satisfies $\tilde{\beta}^{\prime \prime}=c s_{1}^{-\gamma} f(\tilde{\beta}), \tilde{\beta}\left(s_{0}\right)=\varepsilon, \tilde{\beta}\left(s_{1}\right)=\tilde{\beta}^{\prime}\left(s_{1}\right)=0$ and $\tilde{\beta} \leq \varepsilon$. Using $s_{1}^{-\gamma} \leq s^{-\gamma}$ for $s \in\left(s_{0}, s_{1}\right)$ we infer $(2.5)$ and the claim follows. This concludes the proof.

Observe that in the above proof we did not actually use the fact that the domain in which the equation $\Delta u=f(u)$ is set is a half-space. In the existence part of the proof we only used that we can find a ball outside the domain, whereas in the non-existence part we only used that the domain contains a sufficiently large ball. In other words, we have actually proved the following result.

Theorem 2. Suppose (KO) holds, and $f(0)=0, f(t)>0$ for $t>0$ is monotone increasing. Assume $\Omega$ is such that $\bar{\Omega} \neq \mathbb{R}^{N}$ and for each $R>0$ there exists a ball with radius $R$ included in $\Omega$. Then the equation $\Delta u=f(u)$ has a positive solution 
in $\Omega$ if and only if $\int_{0}^{1} \frac{d s}{\sqrt{F(s)}}=\infty$. If this hypothesis is not satisfied, even positive subsolutions do not exist in $\Omega$.

Proof of Theorem 1, case $t_{0}=-\infty$.

Existence. Assume (HS) holds, that is, $\int_{-\infty} f$ is convergent. Fix a positive constant $C^{*}$ such that $C^{*}>2 \int_{-\infty}^{0} f(t) d t=-2 F(-\infty)$. We define implicitly $v$ as follows:

$$
\int_{v(t)}^{0} \frac{d s}{\sqrt{2 F(s)+C^{*}}}=t .
$$

Observe that $v$ is well defined by (HS) and the choice of $C^{*}$. Moreover, by differentiating

$$
-v^{\prime}(t)=\sqrt{2 F(v(t))+C^{*}}
$$

and therefore $v^{\prime}$ is strictly negative and bounded for $t \geq 0$. By differentiating once more we see that $v$ satisfies $v^{\prime \prime}=f(v)$ in $(0,+\infty)$ and thus $u(x)=v\left(x_{N}\right)$ is a (one-dimensional and negative) solution of $(\mathrm{P})$ in the half-space.

Nonexistence. Let us assume that (KO) holds, $\int_{-\infty} f$ is divergent and there exists a sub-solution $u$ of $(\mathrm{P})$ in $\mathbb{R}_{+}^{N}$.

First we observe that

$$
\lim _{x_{N} \rightarrow+\infty} u(x)=-\infty \quad \text { uniformly for } x^{\prime} \in \mathbb{R}^{N-1},
$$

where $x=\left(x^{\prime}, x_{N}\right)$.

In fact, if this were not the case, there would exist $\gamma<0$ and a sequence $x^{(n)}$ with $x_{N}^{(n)} \rightarrow \infty$ such that $u\left(x^{(n)}\right) \geq \gamma$ as $n \rightarrow \infty$. As we observed before the proof of Theorem 1, we may use the condition (KO) to ensure that there exists a radial solution $w_{n}$ of the equation $\Delta w=f(w)$ in $B_{R}\left(x^{(n)}\right)$, with $w_{n}\left(x^{(n)}\right)=2 \gamma$ and $w_{n}=+\infty$ on $\partial B_{R}\left(x^{(n)}\right)$, for some sufficiently large and fixed $R>0$. Observe that $B_{R}\left(x^{(n)}\right) \subset \mathbb{R}_{+}^{N}$ for large $n$, therefore we can apply the comparison principle and deduce $u(x) \leq w_{n}(x)$ in $B_{R}\left(x^{(n)}\right)$ for large $n$. We obtain a contradiction since $w_{n}\left(x^{(n)}\right)=2 \gamma<\gamma \leq u\left(x_{n}\right)$.

By the above argument, and replacing if necessary the half-space $\left\{x_{N}>0\right\}$ by the half-space $\left\{x_{N}>A\right\}$ for some conveniently chosen $A>0$, we can assume that $u<0$ in $\mathbb{R}_{+}^{N}$. Moreover, for a given $M>0$ there exists $R=R(M)>0$ such that $u \leq-M$ on $\mathbb{R}^{N-1} \times\{R\}$ and $R(M) \rightarrow+\infty$ as $M \rightarrow+\infty$.

For any $R, T>0$ consider the finite cylinder

$$
D_{T, R}:=\left\{x=\left(x^{\prime}, x_{N}\right) \in \mathbb{R}_{+}^{N}:\left|x^{\prime}\right|<T, x_{N}<R\right\} .
$$

Let $\Omega_{n}$ be a sequence of smooth bounded domains such that $D_{n, R} \subset \Omega_{n} \subset D_{n+1, R}$, and let $h_{n}$ be a continuous function defined on $\partial \Omega_{n}$ such that $-M \leq h_{n} \leq 0$ on $\partial \Omega_{n}$, $h_{n}=-M$ on $\partial \Omega_{n} \cap\left\{\left|x^{\prime}\right|<n, x_{N}=R\right\}$ and $h_{n}=0$ on $\partial \Omega_{n} \cap\left\{x_{N}<R\right\}$. Consider the problem

$$
\begin{cases}\Delta w=f(w) & \text { in } \Omega_{n} \\ w=h_{n} & \text { on } \partial \Omega_{n}\end{cases}
$$


By the construction of $h_{n}$ we see that $u$ is a subsolution of this problem, while $\bar{u}=0$ is a supersolution (recall that $f(0)>0$ ), and they are ordered. Therefore there exists a solution $w_{n}$ of (2.7) verifying $u \leq w_{n} \leq 0$ in $\Omega_{n}$. Again by the usual elliptic estimates the sequence $w_{n}$ is bounded in $W^{2, p}(K)$ for each compact $K \subset\left\{0 \leq x_{N} \leq R\right\}$. Therefore we can pass to the limit by using a diagonal argument, to obtain a solution $w_{M}$ of the following problem in a strip

$$
\begin{cases}\Delta w=f(w) & \text { in }\left\{0 \leq x_{N} \leq R\right\} \\ w=0 & \text { on } x_{N}=0 \\ w=-M & \text { on } x_{N}=R\end{cases}
$$

Of course we also have $u \leq w_{M} \leq 0$. We claim that actually $w_{M}$ is the maximal solution of $(2.8)$ in the order interval $[u, 0]$. To show this, let $\tilde{w}$ be any solution of (2.8) such that $u \leq \tilde{w} \leq 0$. Therefore $\tilde{w}$ is a subsolution of (2.7), so by comparison $\tilde{w} \leq w_{n}$ in $\Omega_{n}$ for every $n$. At the limit $\tilde{w} \leq w_{M}$ and maximality follows.

By maximality, $w_{M}$ depends only on the variable $x^{N}$ (since any translation of $w_{M}$ in a direction parallel to $\left\{x_{N}=0\right\}$ is still a solution of (2.8)). This means that $v_{M}\left(x_{N}\right)=w_{M}(x)$ is a classical solution of $v_{M}^{\prime \prime}=f\left(v_{M}\right)$ in $(0, R)$ with $v_{M}(0)=0$ and $v_{M}(R)=-M$. We can pass to the limit as $M \rightarrow+\infty$ as before to obtain that $v_{M_{n}} \rightarrow v$ in $C_{\mathrm{loc}}^{2}[0,+\infty)$ for some sequence $M_{n} \rightarrow+\infty$, where

$$
v^{\prime \prime}=f(v) \text { in }(0,+\infty)
$$

with $v(0)=0$. Now $w(x)=v\left(x_{N}\right)$ is itself a solution of $(\mathrm{P})$, and hence, by (2.6), we have $v\left(x_{N}\right) \rightarrow-\infty$ as $x_{N} \rightarrow \infty$.

However, as we already observed, $\left(v^{\prime}\right)^{2}=2 F(v)+v^{\prime}(0)^{2}$ and so $F\left(v\left(x_{N}\right)\right)$ is bounded from below as $x_{N} \rightarrow \infty$, while on the other hand the failure of (HS) means precisely that $F(t)=-\int_{t}^{0} f$ tends to $-\infty$ as $t \rightarrow-\infty$. The proof is concluded.

Acknowledgements. J. G-M and A. Q. were partially supported by Ministerio de Ciencia e Innovación under grant MTM2011-27998 (Spain), A. Q. was partially supported by Fondecyt Grant No. 1110210 and CAPDE, Anillo ACT-125 Chile). All three authors were partially supported by Programa Basal CMM, U. de Chile.

\section{REFERENCES}

[1] S.N. Armstrong, B. Sirakov, Nonexistence of positive supersolutions of elliptic equations via the maximum principle. Comm. Partial Differential Equations 36 (2011), 2011-2047.

[2] C. Bandle, M. Essen, On positive solutions of Emden equations in cone-like domains. Arch. Rational Mech. Anal., 112 (4) (1990), 319-338.

[3] H. Berestycki, I. Capuzzo-Dolcetta, L. Nirenberg, Superlinear indefinite elliptic problems and nonlinear Liouville theorems. Topol. Methods Nonlinear Anal, 4 (1) (1994), $59-78$.

[4] H. Berestycki, L. Caffarelli, L. Nirenberg, Further qualitative properties for elliptic equations in unbounded domains, Ann. Scuola Norm. Sup. Pisa, 25 (1997), 69-94.

[5] Z. Chen, C.-S. Lin, W. Zou, Monotonicity and nonexistence results to cooperative systems in the half space, J. Funct. Anal. 266 (2014), 1088-1105.

[6] M.G. Crandall, H. Ishit, P.-L. Lions, User's guide to viscosity solutions of second-order partial differential equations, Bull. Amer. Math. Soc. 27 (1992) (1), 1-67. 
[7] N. DAncer, Some notes on the method of moving planes, Bull. Austral. Math. Soc. 46 (1992), 425-434.

[8] Y. Du, Order structure and topological methods in nonlinear partial differential equations, Series in PDE and Applications, World Scientific (2006).

[9] A. FARINA, Liouville-type theorems for elliptic problems, in "Handbook of Differential Equations: Stationary Partial Differential Equations", Vol. 4 (Michel Chipot, Editor) (2007), 483-591.

[10] P. Felmer, M. Montenegro, A. QuaAs, A note on the strong maximum principle and the compact support principle, J. Differential Equations 246 (2009), 39-49.

[11] D. Gilbarg, N. Trudinger, Elliptic partial differential equations of second order, SpringerVerlag, 2nd edition, Berlin-Heidelberg, 2001.

[12] J. B. Keller, On solutions of $\Delta u=f(u)$, Comm. Pure Appl. Math. 10 (1957), 503-510.

[13] V. Kondratiev, V. Liskevich, V. Moroz, Positive solutions to superlinear second-order divergence type elliptic equations in cone-like domains. Ann. Inst. H. Poincare Anal. Non Lineaire, 22 (1) (2005), 25-43.

[14] M. Marcus, L. VÉron, Existence and uniqueness results for large solutions of general nonlinear elliptic equations, J. Evol. Equ. 3 (2004), 637-652.

[15] R. Osserman, On the inequality $\Delta u \geq f(u)$, Pac. J. Math. 7 (1957), 1641-1647.

[16] P. Pucci, J. Serrin, The maximum principle. Progress in Nonlinear Differential Equations and their Applications, 73. Birkhauser Verlag, Basel, 2007.

[17] V. RǍDulEscu, Singular phenomena in nonlinear elliptic problems: from boundary blow-up solutions to equations with singular nonlinearities, in "Handbook of Differential Equations: Stationary Partial Differential Equations", Vol. 4 (Michel Chipot, Editor) (2007), 483-591.

[18] J. L. VÁzQUEz, A strong maximum principle for some quasilinear elliptic equations, Appl. Math. Optim. 12 (3) (1984), 191-202.

\section{J. García-Melián}

Departamento de AnÁlisis Matemático, Universidad de La Laguna. C/. Astrofísico Francisco SÁnchez s/n, 38271 - LA Laguna, SPAIN and

Instituto Universitario de Estudios Avanzados (IUdEA) en Física AtómicA, Molecular y Fotónica, Universidad de La LAguna

C/. Astrofísico Francisco SÁnchez s/n, 38203 - La Laguna , SPAIN

E-mail address: jjgarmel@ull.es

\section{A. QUAAS}

Departamento de Matemática, Universidad Técnica Federico Santa María

Casilla V-110, Avda. España, 1680 - VAlparaíso, CHILE.

E-mail address: alexander.quaas@usm.cl

B. SIRAKOV

Departamento de Matemática, PUC-Rio

Rua Marques de SÃo Vicente, 225, Rio de Janeiro - RJ, CEP 22451-900, BRASiL.

E-mail address: bsirakov@mat.puc-rio.br 